\title{
Unexpected location of pacemaker electrode: Is it right?
}

\section{Ubicación inesperada del electrodo marcapasos: ¿es correcto?}

\author{
Juan F. Vasquez-Rodriguez ${ }^{1 *}$, Ramón Medina-Mur ${ }^{1}$, Lukas Salazar ${ }^{1}$, Daniel Isaza ${ }^{1,2}$, and \\ Claudia P. Jaimes ${ }^{1,3}$ \\ ${ }^{1}$ Department of Cardiology; ${ }^{2}$ Coronary Critical Care Unit, Division of Cardiology; ${ }^{3}$ Department of Cardiovascular Imaging, Division of Cardiology, \\ Fundación Cardioinfantil - Instituto de Cardiología, Bogotá D.C, Colombia
}

A 74-year-old woman presented with weakness, dizziness, dyspnea, and chest pain. A $2^{\text {nd }}$ degree atrioventricular block was found. Transvenous temporary pacemaker (TTP) was implanted. Post-TTP implantation electrocardiography (ECG) showed a paced rhythm with a right branch block (RBBB) morphology, inconsistent with the right ventricular stimulation - as expected for this type of device. Chest X-ray showed the lead entering the right atrium through the superior vena cava, but the tip directed toward the left ventricular apex (Fig. 1). ECG pattern and location on chest $X$-ray suggested a left ventricle (LV) location of the electrode.

Transthoracic echocardiogram (TTE) documented a patent foramen ovale (PFO). The TTP lead crossed the
PFO into the left atrium, and its distal segment formed a loop in the LV apex (Fig. 2).

TTP insertion is usually performed using ultrasound-guided venous punctures, but with blind electrode positioning. This procedure is considered safe and few complications are seen like as non-pericardial bleeding (2.4\%), cardiac tamponade $(0.6 \%)$, and iatrogenic pneumothorax $(2.4 \%)^{1}$. Unnoticed stimulation of LV after a venous puncture is infrequent. The available data highlight the fortuitous passage of the lead through interatrial septum defects as a possible cause. Other possibilities include left ventricular location due to interventricular septum (IVS) perforation; RV apical location with depolarization vector going from left to right, in dilated cardiomyopathy, secondary to left

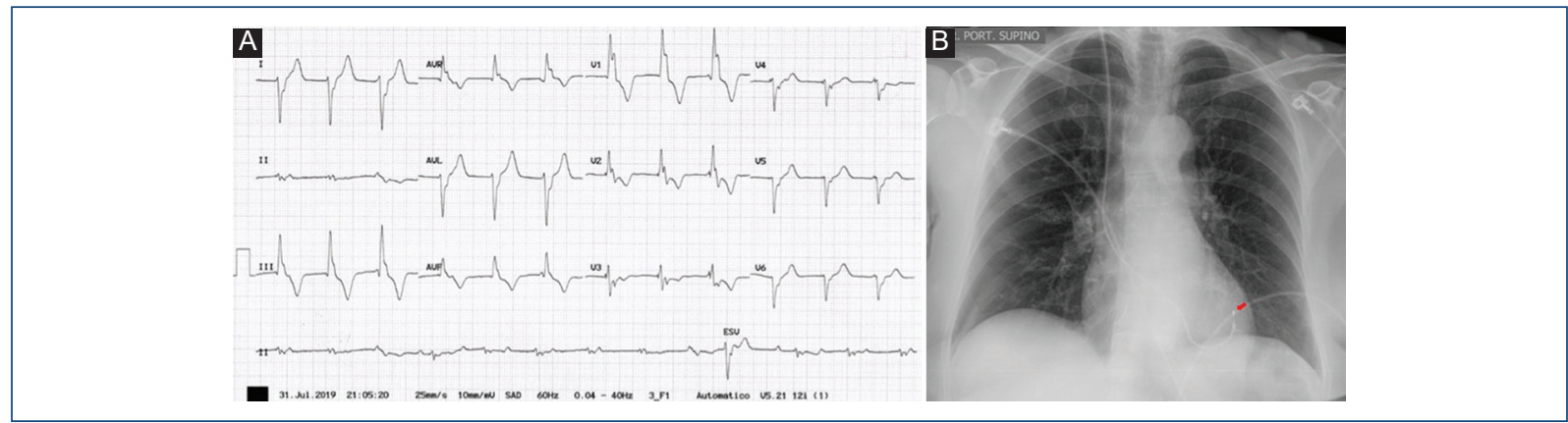

Figure 1. A: ECG: paced rhythm with a right branch block morphology. B: chest X-ray: lead in the right atrium with its tip directed toward the left ventricular apex (red arrow).

\section{Correspondence:}

*Juan F. Vasquez-Rodriguez

E-mail: juanfevasquez8@gmail.com
Date of reception: 11-04-2020

Date of acceptance: 18-06-2020

DOI: 10.24875/ACME.M21000214
Available online: 12-11-2020

Arch Cardiol Mex (Eng). 2021;91(2):212-213

www.archivoscardiologia.com 2604-7063 / C 2020 Instituto Nacional de Cardiología Ignacio Chávez. Published by Permanyer. This is an open access article under the CC BY-NC-ND license (http://creativecommons.org/licenses/by-nc-nd/4.0/). 


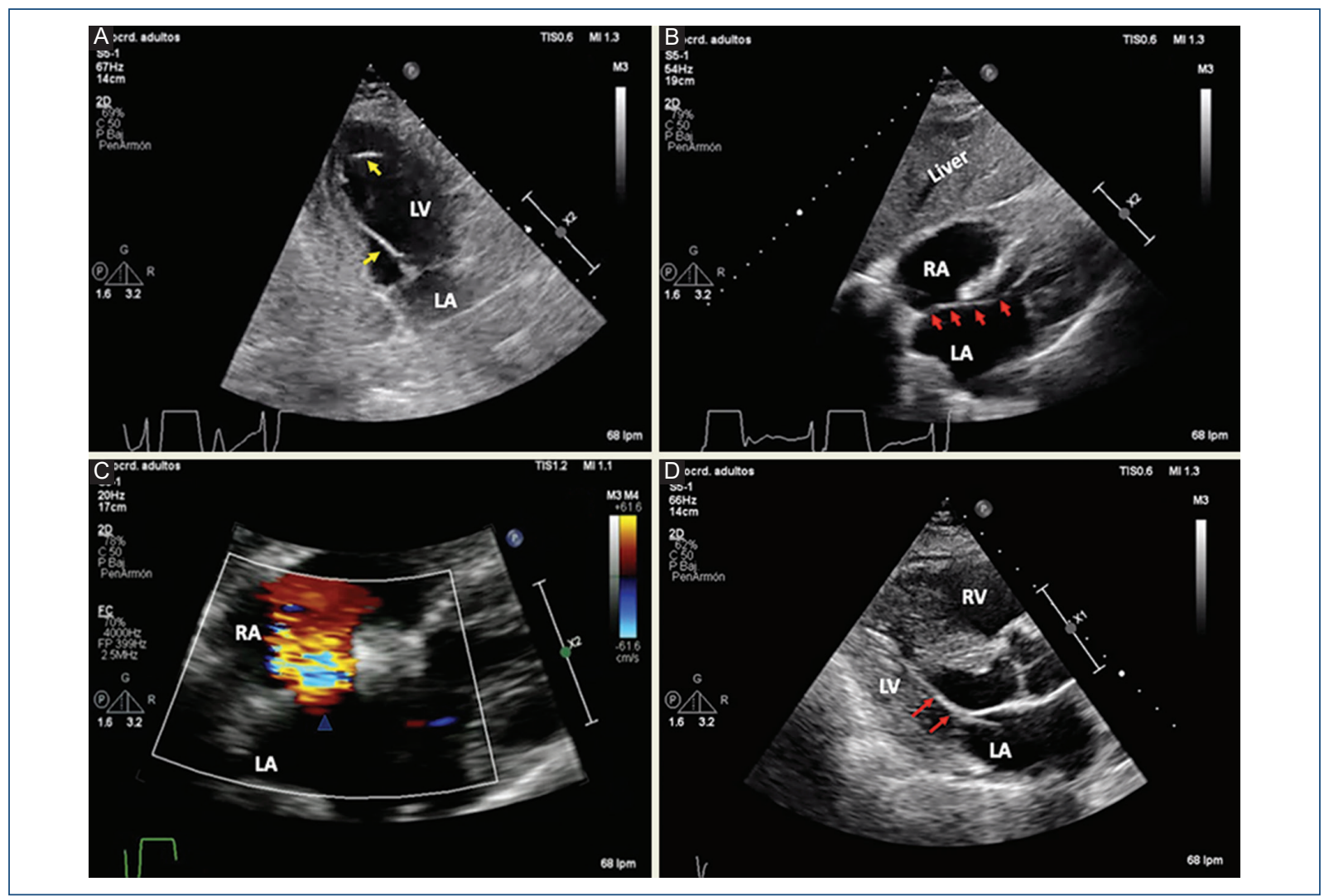

Figure 2. A: apical 2 chamber view: TTP lead forming a loop in the left ventricle apex (yellow arrow). B: subcostal 4 chamber view: TTP lead crossing through a PFO (red arrow). C: subcostal 4 chamber view: color Doppler at the interatrial septum (blue arrowhead). D: parasternal long-axis view: TTP lead in the left ventricle (red arrow). LA: left atria, LV: left ventricle, RA: right atria, RV: right ventricle.

displacement of the IVS and RV; and epicardial stimulation due to RV free wall perforation or by accessing LV coronary venous tributaries through the coronary sinus with presence of QS in D1 and aVL. The left location of these devices is a potential cause of embolism².

A pacemaker stimulation with RBBB morphology after TTP insertion could help to predict the electrode location. Complementary studies such as chest X-ray, TTE, and fluoroscopy are useful to confirm the correct positioning of the electrode. The latter being less accessible to the patient's bedside, but allowing the correct lead placement.

\section{Funding}

This research did not receive any specific grant from funding agencies in the public, commercial, or not-forprofit sectors.

\section{Conflicts of interest}

None.

\section{Ethical disclosures}

Protection of human and animal subjects. The authors declare that no experiments were performed on humans or animals for this study.

Confidentiality of data. The authors declare that they have followed the protocols of their work center on the publication of patient data.

Right to privacy and informed consent. The authors declare that no patient data appear in this article.

\section{References}

1. Metkus TS, Schulman SP, Marine JE, Eid SM. Complications and outcomes of temporary transvenous pacing: an analysis of $>360,000$ patients from the national inpatient sample. Chest. 2019;155:749-57.

2. Gelder BM, Bracke FA, Oto A, Yildirir A, Haas PC, Seger JJ, et al. Diagnosis and management of inadvertently placed pacing and ICD leads in the left ventricle: a multicenter experience and review of the literature. Pacing Clin Electrophysiol. 2000;23:877-83. 